\title{
Escala de Atitude Frente à Fofoca: Evidências de Validade e Confiabildade
}

Attitudes Toward Gossip Scale: Evidences of Validity and Reliability

Escala de Actitud ante el Chisme: Evidencias de Validad y Confiabilidad

Valdiney V. Gouveia,

Deliane Macedo Farias de Sousa,

Andréa Xavier de Albuquerque-Souza,

Roseane Christhina da Nova Sá-Serafim \& Clênia Maria Toledo de Santana Gonçalves

Universidade Federal da Paraíba

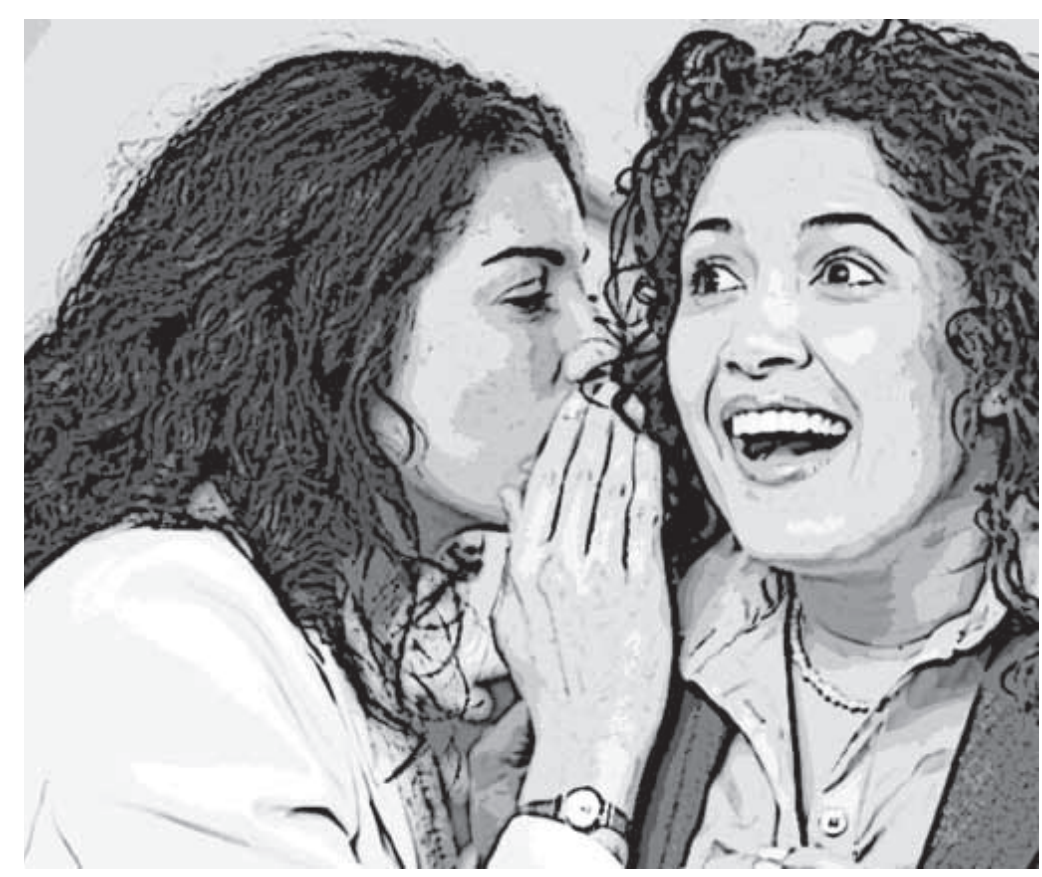


Resumo: A fofoca é um elemento comum nas conversas cotidianas tanto de adultos quanto de crianças, está presente em todas as culturas e explica diversos comportamentos sociais. Portanto, considerando a relevância desse construto e a ausência de medida específica no contexto brasileiro, decidiu-se adaptar a Escala de Atitudes frente à Fofoca (EAFF) para, especificamente, conhecer evidências de sua validade fatorial e de sua consistência interna. O instrumento é formado por 12 itens, respondidos em escala de 5 pontos, e mede dois fatores: valor moral e valor social da fofoca. Participaram da pesquisa 261 estudantes universitários de instituições públicas e privadas de João Pessoa (PB), com idade média de 26 anos, sendo a maioria do sexo feminino (71,6\%). Por meio de análises fatoriais confirmatórias, foram testados dois modelos: (1) unifatorial (alternativo) e (2) bifatorial (modelo original). Os resultados mostraram que o modelo bifatorial apresentou os melhores indicadores de ajuste, com índices de confiabilidade (Alfa de Cronbach) aceitáveis para fins de pesquisa.

Palavras-chave: Fofoca. Atitude. Escalas. Comportamento social.

Abstract: Gossip is a common element in both adults and children daily conversation and is present in all cultures, accounting for several social behaviors. So, considering the relevance of this construct and the lack of a specific measure in the Brazilian context, the Attitudes Toward Gossip Scale (ATG) was adapted for this purpose. Specifically, this work aimed to know evidences of its factorial validity and its reliability. This instrument consists of 12 items rated on a 5-point scale, assessing two factors: the moral value and the social value of gossip. The participants were 261 undergraduate students from public and private institutions from João Pessoa (PB, Brazil), with the mean age of 26 years old, most of them females $(71.6 \%)$. Two models were tested by confirmatory factor analysis: (1) one-factor (alternative model) and (2) twofactor (original model). Results pointed that the two-factor model showed the better fit indexes, with acceptable reliability (Cronbach's Alpha) for research purposes.

Keywords: Gossip. Attitude. Scales. Social beahvior.

Resumen: El chisme es un elemento común en las conversaciones cotidianas tanto de adultos como de niños, y se encuentra presente en todas las culturas, explicando distintos comportamientos sociales. Por lo tanto, considerando la relevancia de este constructo y la ausencia de medida específica en el contexto brasileño, se ha decidido adaptar la Escala de Actitudes ante el Chisme (EAFF). Específicamente, se ha tenido como objetivo conocer evidencias de su validad factorial y consistencia interna. Ésta está conformada por 12 ítems, respondidos en una escala de 5 puntos, y mide dos factores: el valor moral y el valor social del chisme. Han participado de la investigación 261 estudiantes universitarios de instituciones públicas y privadas de João Pessoa (PB), con una edad promedio de 26 años, siendo la mayoría del sexo femenino (71,6\%). A través de análisis factoriales confirmatorias se han testado dos modelos: (1) unifactorial (alternativo) y (2) bifactorial (modelo original). Los resultados han demostrado que el modelo bifactorial ha presentado los mejores indicadores de ajuste, con índices de confiabilidad (Alfa de Cronbach) aceptables para los fines de investigación.

Palabras clave: Chisme. Actitud. Escalas. Conducta social.

O presente estudo contou com o apoio do $\mathrm{CNPq}$ e da CAPES por meio de bolsas de produtividade em pesquisa e doutorado concedidas ao primeiro e ao segundo autores, respectivamente. Os autores agradecem a essas duas instituições.
Pesquisas transculturais e informações históricas sugerem que a disposição à fofoca está no cerne das relações sociais desde os primórdios da humanidade (Barkow, 1992; Kniffin \&Wilson, 2005; Paine, 1967; Schein, 1994). É possível pensar que essa disposição, além de educar o ouvinte sobre as normas e regras sociais, é uma ferramenta eficaz para transmitir informações sobre códigos culturais e orientações sobre como viver em grupo, preocupando mais ou atraindo maior atenção para condutas que rompam normas sociais. Por exemplo, de acordo com Baumeister, Zhang e Vohs (2004), as pessoas estão mais interessadas em ouvir ou dizer coisas ruins sobre os outros do que coisas boas.

Foster assinala que:

para funcionar eficientemente em
um ambiente social complexo, os
seres humanos requerem informações


sobre aqueles que os rodeiam. Mas interconexões sociais são complexas, e é impossível estar presente em muitos intercâmbios para reunir essas informações diretamente. Assim, muitas pessoas ficam ansiosas para buscá-las através de um intermediário, não se dando ao trabalho de confirmá-las posteriormente, quer de forma direta, quer indireta. Esse fenômeno é claramente denominado fofoca, sendo um comportamento social importante que quase todos experimentam, e presumivelmente entendem (2004, p.78)

Apesar de a palavra fofoca ser entendida popularmente como conversa fiada, sua transmissão é considerada importante para o estabelecimento de amizades, uma vez que está presente nas conversas do dia a dia. A propósito, Noon e Delbridge (1993) defendem a ideia de que a palavra é frequentemente usada para comunicar muitos significados alternativos. Não é por menos que os pesquisadores interessados em estudar esse construto o consideram relevante para a manutenção das relações sociais, uma vez que permite o intercâmbio de conhecimentos e até de entretenimento mútuo. Em outras palavras, a fofoca é a maneira de obter informações sobre os outros que pode orientar sobre como a pessoa precisa conduzir sua própria vida ou garantir maior autoestima por meio de comparação com os demais (Levin \& Arluke, 1987).

Pode-se, desse modo, dizer que fofocar é uma forma importante de comunicação e de aprendizagem social que serve para unir as pessoas e para compartilhar informações sobre si e sobre os outros. Nesse contexto, Dunbar (1996) destaca duas funções para a fofoca: a primeira visa a fortalecer o vínculo afetivo entre narrador e ouvinte à medida que passam o tempo conversando e trocando informações de interesse mútuo, e a segunda diz respeito ao fato de que as informações contidas na fofoca podem ser úteis para aprender sobre a pessoa e para melhorar a relação, uma vez que se supõe que a pessoa-alvo seja alguém da esfera social do ouvinte.

Segundo Baumeister, Zhang e Vohs (2004) um processo de aprendizagem de todas as regras e orientações de como viver em uma cultura implica socialização de conhecimentos, informações, crenças e valores. Concomitantemente, esses autores indicam que as fofocas revelam informações potencialmente úteis sobre a cultura, bem como sobre o funcionamento da sociedade, pois elas permitem aprender, por observação, com os triunfos e as desventuras das pessoas, e verificar qual é sua percepção imediata. Essa perspectiva ajuda a explicar alguns achados sobre a fofoca, como o que indica que nem sempre ela é depreciativa, e que as pessoas, às vezes, fofocam sobre estranhos. De fato, Sabini e Silver (1982) comentam que a fofoca envolve essencialmente códigos de conduta e normas morais incorporados em histórias concretas. Nesse sentido, constitui uma aprendizagem pautada em observação, e permite que, ao ouvir as desgraças dos outros, a pessoa evite cometer os mesmos erros.

Partindo-se do ponto de vista de que a fofoca existe como forma de adquirir informação, na perspectiva evolutiva, seria possível prever que as pessoas estivessem mais interessadas na informação que pudesse ser explorada para o ganho social. Segundo McAndrew e Milenkovic (2002), espera-se encontrar maior interesse em informações negativas (por exemplo, desgraças, escândalos) sobre pessoas de alto poder aquisitivo, status e potenciais rivais, pois isso seria algo que poderia ser explorado. Informações negativas sobre pessoas socialmente inferiores não têm utilidade prática. Também deve haver menos interesse em repassar informações negativas sobre aliados (amigos e parentes) do que sobre pessoas percebidas como não aliadas. Por outro lado, informações 
positivas (súbita elevação de status) sobre os aliados têm muito mais probabilidade de serem espalhadas.

A ênfase dada à fofoca tende a variar segundo a área de estudo. Kniffin e Wilson (2005), por exemplo, afirmam que antropólogos consideram historicamente a fofoca como uma ferramenta que serve para a manutenção dos grupos, enquanto psicólogos tendem a considerar o seu uso como uma atitude (positiva e negativa) em detrimento dos interesses individuais. Seja como for, parece haver suficiente acordo de que a fofoca compreende uma discussão avaliativa entre as pessoas que estão familiarizadas mutuamente sobre os assuntos pessoais de uma terceira que não está presente (Eder \& Enke, 1991; Sabini \& Prata, 1982). Contudo, uma definição operacional da fofoca tem sido difícil de delinear, pois dizer que uma afirmação é negativa depende do contexto; muitas vezes, avaliações sutis são incorporadas em um tom alto-falante ou nas brincadeiras que os estrangeiros não conseguem entender, e isso dificulta conceituar o que é ou não fofoca (Wert \& Salovey, 2004).

Comumente, existem tópicos que são mais atrelados ou passíveis de fofocas. Nesse sentido, os seguintes têm sido mais mencionados: qualidades pessoais e idiossincrasias, surpresas comportamentais e inconsistências, falhas de caráter, discrepâncias entre o comportamento real e as reivindicações morais, maus modos, modos de comportamento socialmente inaceitáveis, defeitos, impropriedades, omissões, presunções, erros condenáveis, desgraças e fracassos (Bergmann, 1993; Wert \& Salovey, 2004).

Pode-se admitir que a percepção social é frequentemente afetada pela fofoca, já que os seres humanos gastam parcela significativa de suas interações participando, de uma ou de outra forma, de fofocas (Foster, 2004). Assim, de acordo com a intensidade e a direção (positiva e negativa), a fofoca gera impacto social em vários níveis de organização, e pode mudar no espaço e no tempo. A partir das explicações antes mencionadas, compreende-se que, independentemente da perspectiva teórica, o ato de fofocar está a serviço do grupo; espera-se que, quanto maior a aceitação e a partilha da veiculação do assunto (tema de fofoca) em relação a outrem, maior o poder de controle social por parte do grupo que propaga a fofoca.

Com relação à forma como vem sendo estudada a fofoca, as pesquisas têm raízes em diversas disciplinas, contribuindo para uma variedade substancial de métodos de investigação. Foster fez uma análise e descrição dos métodos mais comumente utilizados, de seus benefícios e inconvenientes, e concluiu que os mais frequentes foram: observações participantes, gravação com uso de áudio e vídeo, escuta em lugares públicos (eavesdropping), questionários de levantamentos e pesquisas experimentais. No que se refere à abordagem de estudo, o tema fofoca tem sido tratado de forma descritiva nas notas etnográficas (Acheson, 1988), explicado pelos evolucionistas principalmente por seus benefícios individuais (McAndrew \& Milenkovic, 2002) ou reconhecido como resistente à pesquisa sistemática (Noon \& Delbrige, 1993).

O antes comentado não implica dizer que a fofoca não tenha sido objeto de pesquisa. Nessa direção, de acordo com Foster, (2004), é possível encontrar muitas pesquisas a respeito, sobretudo nas ciências humanas e sociais. Porém, o assunto é pouco conhecido no contexto da Psicologia, segundo buscas realizadas no dia 8 de fevereiro de 2010. Especificamente, no âmbito internacional, ao introduzir no site de busca da American Psychological Association - APA PsycNET ${ }^{\mathrm{TM}}$ 
o termo gossip como descritor, foram obtidos 24 resultados, sendo 19 artigos e cinco livros. Com o uso do termo correspondente em português (fofoca) no índice de assuntos do Portal de Periódicos Eletrônicos de Psicologia (PEPSIC), surgiu apenas um registro. Repetindo essa busca no Scientific Eletronic Library Online SCIELO e restringindo-a para o Brasil, não foi encontrado qualquer registro. Ampliando-se a busca para o Google Acadêmico com o mesmo termo (em qualquer lugar do artigo), identificaram-se 195 ocorrências, contudo, nenhuma considerou a fofoca como tema central.

Conclui-se, portanto, que muito há ainda que estudar a respeito do assunto no contexto nacional. Desse modo, um passo preliminar e crucial nessa direção será contar com uma medida adequada sobre esse construto. Procurando a respeito, ao menos dois instrumentos foram encontrados: Questionário de Tendência a Fofocar - QTF (Nevo, Nevo, \& Derech-Zehavi, 1994) e Escala de Atitudes Frente à Fofoca - EAFF (Litman \& Pezzo, 2005). Considerando que esta última é uma medida com poucos itens e parâmetros psicométricos satisfatórios, decidiu-se adaptá-la ao contexto brasileiro, e, com essa finalidade, demanda-se inicialmente descrevê-la.

\section{Escala de Atitudes Frente à Fofoca - EAFF}

Originalmente elaborada nos Estados Unidos por Litman e Pezzo (2005), essa medida contava com 29 itens que descreviam atitudes sobre a natureza geral da fofoca, sendo respondidos em escala de 5 pontos, que variavam de 1 (discordo totalmente) a 5 (concordo totalmente). Seus autores descrevem quatro estudos que embasaram sua elaboração e as evidências de sua validade e consistência interna:
Estudo 1. Refinamento da versão experimental. A versão inicial foi respondida por 644 estudantes de Psicologia, com idades entre 18 e 22 anos. Os dados foram submetidos à análise fatorial dos eixos principais (PAF, rotação promax), procurando diferenciar as estruturas fatoriais para homens e mulheres. Essa análise sugeriu a possibilidade de dois ou três fatores serem extraídos em ambos os grupos. Na solução com três fatores, os dois primeiros foram praticamente idênticos aos observados naquela com dois fatores. Contudo, o terceiro fator foi formado por itens diferentes para homens e mulheres, apresentando significado de difícil interpretação, o que resultou em sua eliminação; foi adotada, portanto, a solução bifatorial, que reuniu 12 itens igualmente distribuídos nos fatores valor moral (VM) e valor social (VS), com Alfas de Cronbach de 0,75 e 0,76, respectivamente.

Estudo 2. Comprovação da estrutura bifatorial. Esse estudo procurou testar a estrutura fatorial mais adequada para a EAFF. Participaram 170 estudantes de Psicologia, sendo 87 mulheres e 83 homens, que responderam à versão desse instrumento com 12 itens. A fim de identificar a melhor solução fatorial, realizaram-se múltiplas análises fatoriais confirmatórias adotando o estimador ML (máxima verossimilhança). Quatro modelos foram testados: Modelo $A_{\llcorner}$que previa um único fator e que reunia os 12 itens; Modelo B, idêntico ao anterior, mas que incluía também um fator de erro de medida saturando nos 12 itens; Modelo $C_{L}$ com os dois fatores previstos (VM e VS), com seis itens saturando em cada um, e Modelo $D_{L}$ que previa os mesmos dois fatores, mas também um fator geral de erro de medida, como no Modelo B. Como era teoricamente esperado, o modelo bifatorial (Modelo C) apresentou os melhores indicadores de ajuste: $\chi^{2}(53)=101,75, \chi^{2} / \mathrm{gl}=1,92$, GFI $=0,91, C F I=0,92, N N I=0,90$ e RMSEA = 0,07. Os Alfas de Cronbach para os fatores 
específicos e a escala como um todo foram superiores a 0,70 .

Estudo 3. Evidências de validade de critério. Seus proponentes procuraram relacionar as subescalas da EAFF com dois instrumentos referentes ao construto de interesse: questionário de tendência a fofocar (QTF) e cenários de fofoca. Participaram 442 estudantes de Psicologia (342 mulheres e 100 homens), com idades entre 18 e 40 anos. Os resultados indicaram que as pontuações nos fatores valor social (VS) e valor moral (VM) se correlacionaram positivamente com aquelas no $Q T F$ ( $r=0,60$ e 0,37, respectivamente). Quanto aos cenários de fofoca, quando a fofoca era apresentada como positiva, não houve correlação entre os fatores da EAFF com a intenção e o interesse em transmitir a fofoca; porém, quando a fofoca tinha uma conotação negativa, foram observadas correlações positivas dos fatores VS e VM com a intenção ( $r=0,50$ e 0,20, respectivamente) e o interesse $(r=0,40$ e 0,19 , respectivamente) em transmitir a fofoca. Decidiu-se realizar regressões para verificar em que medida os fatores da EAFF explicavam o interesse e a intenção em transmitir fofocas negativas. Os resultados indicaram que unicamente o VS predisse o interesse $\left(R^{2}=0,19 ; \beta=0,08 ; t=3,90, p\right.$ $<0,01)$ e a intenção $\left(R^{2}=0,25 ; \beta=0,12\right.$; $t=6,40, p<0,001)$ de transmitir fofoca negativa.

Estudo 4. Evidências de validade de construto. Nesse caso, procurou-se conhecer a relação da EAFF com medidas relacionadas à fofoca, como instrumentos que avaliam o interesse em interação social (por exemplo, extroversão), traços que podem inibir a socialização (por exemplo, ansiedade) e necessidade de aprovação social. Participaram 268 estudantes de Psicologia, a maioria mulheres $(71,6 \%)$, com idades entre 18 e 43 anos. Além da EAFF e do QTF, foram respondidos os seguintes instrumentos: Escala de Curiosidade
Interpessoal, Escala de Extroversão e Escala de Desejabilidade Social. As pontuações nos fatores VS e VM se correlacionaram com aquelas das medidas QTF ( $r=0,55$ e 0,36, respectivamente; $p<0,01$ para ambos), de curiosidade interpessoal $(r=0,24$ e 0,14 , respectivamente; $p<0,05$ para ambos) e desejabilidade social $(r=-0,30$ e -0,22, respectivamente; $p<0,05$ para ambos); não se observaram correlações significativas com as pontuações em extroversão. Os autores concluíram que, embora tenham observado correlações entre as medidas consideradas, a EAFF se diferencia das outras, pois ela aborda mais diretamente as atitudes frente à fofoca em si enquanto as demais se referem às interações sociais de onde a fofoca emerge.

Em resumo, os resultados descritos por Litman e Pezzo (2005) apoiam sua medida de atitudesfrente à fofoca, mais especificamente, nos fatores valor social e valor moral a ela atribuídos, uma vez que reúnem evidências de validade (fatorial, critério e construto) e consistência interna. Portanto, essa é uma medida adequada quando se deseja estimar a favorabilidade ou não das pessoas frente à fofoca, servindo para orientar o trabalho do psicólogo no contexto socio-organizacional e para possibilitar o seu emprego em pesquisas que visam a conhecer os correlatos de tais atitudes. Nesse contexto, reconhecendo a importância do construto fofoca e levando em conta a escassez de estudos a respeito no contexto brasileiro, como ficou anteriormente demonstrado, decidiu-se adaptar a Escala de Atitudes frente à Fofoca para uso no Brasil, reunindo evidências de sua validade fatorial e consistência interna.

\section{Método}

\section{Participantes}

Contou-se com uma amostra não probabilística, isto é, de conveniência, 
formada por pessoas que, convidadas, concordaram em colaborar. Participaram 261 estudantes universitários de instituições públicas e privadas de ensino de João Pessoa, Paraíba. Estes tinham idades entre 18 e 70 anos $(m=26,1 ; d p=8,7)$, sendo a maioria do sexo feminino $(71,6 \%)$, solteira $(68,8 \%)$ e católica (54\%).

\section{Instrumentos}

Os participantes responderam um caderno contendo três partes principais:

Escala de Atitudes Frente à Fofoca - (EAFF) (Littman \& Pezzo, 2005). Esta compreende 12 itens que cobrem aspectos sociais (por exemplo, fazer fofoca é uma boa forma de quebrar o gelo) e morais (por exemplo, é errado falar sobre outras pessoas quando elas não estão presentes) em relação à fofoca. Tais itens são respondidos em escala de 5 pontos, variando de $1=$ discordo totalmente a $5=$ concordo totalmente. Nenhuma informação é conhecida sobre seus parâmetros psicométricos no contexto brasileiro.

Escala de Desejabilidade Social de MarloweCrowne (EDSMC). Desenvolvida por Crowne e Marlowe (1960), a presente versão compreende uma adaptação brasileira reduzida, formada por 20 itens, proposta por Gouveia, Guerra, Sousa, Santos e Costa (2009). Seus itens expressam a necessidade de aprovação por parte de outras pessoas (por exemplo, se pudesse entrar em um cinema sem pagar e ter certeza de que não seria visto(a), provavelmente eu o faria; sou sempre cuidadoso(a) com meu jeito de vestir). As respostas são dadas em escala dicotômica, com as pontuações 0 (falso) ou 1 (verdadeiro). Segundo os proponentes dessa versão, essa medida é unifatorial, apresentando consistência interna satisfatória $(K R 20=0,76)$.

Informações demográficas. Os participantes foram solicitados a informar os seguintes dados: estado civil, idade, religião e sexo, que tinham o propósito unicamente de caracterizá-los sem, entretanto, identificálos.

A tradução da EAFF do inglês para o português foi efetuada por duas psicólogas bilíngues, e foi submetida à apreciação de um terceiro psicólogo também bilíngue com o fim de avaliar a adequação dos termos para o contexto do estudo. Antes de aplicá-la, procedeu-se a sua validação semântica, considerando um grupo de 24 estudantes universitários que estavam cursando o primeiro período em instituições públicas e privadas de João Pessoa (PB). Os participantes foram solicitados a assinalar os itens que lhes parecessem problemáticos ou ambíguos, sugerindo uma forma alternativa de redigi-los, e também foram solicitados a avaliar as instruções sobre como responder os itens e a escala de resposta a ser utilizada. Não foi verificada qualquer alteração substancial, mantendo-se a versão traduzida consensualmente, que está disponível, sob solicitação, a um dos autores.

\section{Procedimento}

Os participantes responderam ao instrumento individualmente, porém em ambiente coletivo de sala de aula. Três colaboradores devidamente treinados ficaram responsáveis por sua aplicação. Após a autorização do professor da disciplina, estes se apresentavam e solicitavam a colaboração dos estudantes presentes, informando-lhes que se tratava de uma pesquisa sobre as atitudes das pessoas no seu dia a dia, não havendo respostas certas ou erradas. Concordando em participar no estudo, todos precisaram preencher um Termo de Consentimento Livre e Esclarecido. Enfatizou-se o caráter voluntário de sua participação e a confidencialidade dos dados obtidos, seguindo procedimentos éticos em vigor. Em média, 15 minutos foram suficientes para concluir sua participação. 


\section{Análise de dados}

O SPSS (versão 15) e o AMOS (versão 7) foram os programas utilizados para analisar os dados. Com base no primeiro, efetuaram-se cálculos de estatísticas descritivas (medidas de tendência central e dispersão) e Alfa de Cronbach (consistência interna) para os fatores resultantes da escala. Posteriormente, o segundo programa permitiu testar a estrutura fatorial original, composta por dois fatores, contrastando-a com uma alternativa, unifatorial, em que todos os itens foram indicados saturar em um único fator. Considerou-se como entrada a matriz de covariâncias (estimador $M L$, máxima verossimilhança). Os seguintes indicadores de ajuste foram considerados (Garson, 2003): (1) $\chi^{2}$ (Qui-quadrado), que testa se o modelo se ajusta aos dados, com valores altos representando um ajuste inadequado - esse indicador sofre influência do tamanho da amostra, podendo não funcionar com amostras grandes ( $>$ 200); (2) razão entre qui-quadrado e graus de liberdade $\left(\chi^{2} /\right.$ g.l.) do modelo é uma alternativa satisfatória, com valores entre 2 e 3 indicando um ajuste adequado; (3) o índice de adequação de ajuste (Goodness-of-Fit Index - GFI) e o índice de adequação de ajuste ponderado (Adjusted Goodness-of-Fit Index - AGFI), que leva em conta os graus de liberdade do modelo com respeito ao número de variáveis consideradas, são dois indicadores complementares; valores iguais ou superiores a 0,90 são desejáveis como indicação de ajuste do modelo; (4) a RMSEA - Root Mean Square Error Approximation considera os residuais: quanto menor o seu valor, melhor ajuste do modelo, recomendando-se um valor próximo a 0,05, aceitando-se até 0,10 como adequação do modelo, e (5) Comparative Fit Index - CFI, que é um índice comparativo, adicional, de ajuste do modelo, com valores mais próximos de 1 indicando melhor ajuste; admitem-se valores de 0,90 ou mais como expressão de ajuste adequado.

Com o propósito de comparar os modelos rivais, três indicadores são tidos em conta (Garson, 2003): o Expected Cross-Validation Index - ECVI e o Consistent Akaike Information Criterion - CAIC são úteis para a comparação de modelos, considerando-se mais adequado aquele que apresenta valores menores. Contudo, tais indicadores não oferecem uma prova estatística; nesse caso, pode ser relevante considerar a diferença entre os qui-quadrados e respectivos graus de liberdade $\left(\Delta \chi^{2} / g\right)$, penalizando o modelo com maior qui-quadrado.

\section{Resultados}

Evidências de validade fatorial e consistência interna

Foram testados dois modelos para representar a estrutura fatorial da EAFF: o primeiro (Modelo $1, M_{1}$ ) reuniu os 12 itens que foram definidos como saturando em um único fator geral, e o segundo (Modelo 2, $\mathrm{M}_{2}$ ), em que se previam dois fatores (valor moral e valor social), com seis itens saturando em cada um, de acordo com a estrutura apresentada quando da elaboração dessa medida. Os índices de ajuste para cada um desses modelos podem ser observados na Tabela 1.

Tabela 1. Indicadores de ajuste dos modelos uni e bifatorial

\begin{tabular}{cccccccccc}
\hline Modelos & $\chi^{2}$ & gl & $\chi^{2} / g \mid$ & GFI & AGFI & CFI & $\begin{array}{c}\text { RMSEA } \\
(\text { IC90\% })\end{array}$ & CAIC & ECVI \\
\hline $\begin{array}{c}\text { Unifatorial } \\
\left(\mathrm{M}_{1}\right)\end{array}$ & 129,43 & 53 & 2,44 & 0,92 & 0,88 & 0,88 & $\begin{array}{c}0,07 \\
(0,058-0,091)\end{array}$ & 293,54 & 0,70 \\
& & & & & & & & & \\
$\begin{array}{c}\text { Bifatorial } \\
\left(\mathrm{M}_{2}\right)\end{array}$ & 110,62 & 52 & 2,13 & 0,93 & 0,90 & 0,90 & $\begin{array}{c}0,07 \\
(0,049-0,083)\end{array}$ & 281,30 & 0,62 \\
\hline
\end{tabular}


Como pode ser observado nessa tabela, o modelo que apresentou os melhores índices de ajuste foi aquele composto por dois fatores $\left(\chi^{2} / \mathrm{gl}=2,13, \mathrm{GFI}=0,93, \mathrm{AGFI}=90, \mathrm{CFI}=0,90\right.$ e RMSEA = 0,07), como originalmente proposto. Embora a diferença entre os índices de ajuste dos modelos $M_{1}$ e $M_{2}$ não seja tão evidente à primeira vista, a comparação entre seus respectivos qui-quadrados e graus de liberdade a torna mais clara $\left(\Delta \chi^{2}[1]=18,81, p<0,001\right)$. Indicadores complementares de ajuste ( $E C V I$ e CAIC) corroboram a melhor adequação do $\mathrm{M}_{2}$, que assume a estrutura bifatorial da EAFF. Concretamente, esses foram menores para esse modelo. Destaca-se que todas as saturações (lambdas) foram estatisticamente diferentes de zero $(\lambda \neq 0 ; z>1,96$, $p<0,001$ ), com valor médio de 0,51, variando de 0,12 a 0,72. Considerando os IMs (índices de modificação), percebeu-se a necessidade de fixar a correlação entre os erros de medida dos itens 4 (é errado falar sobre os demais quando eles não estão próximos) e 6 (não se pode confiar em fofocas) do fator valores morais. O modelo resultante é apresentado na Figura 1, a seguir.

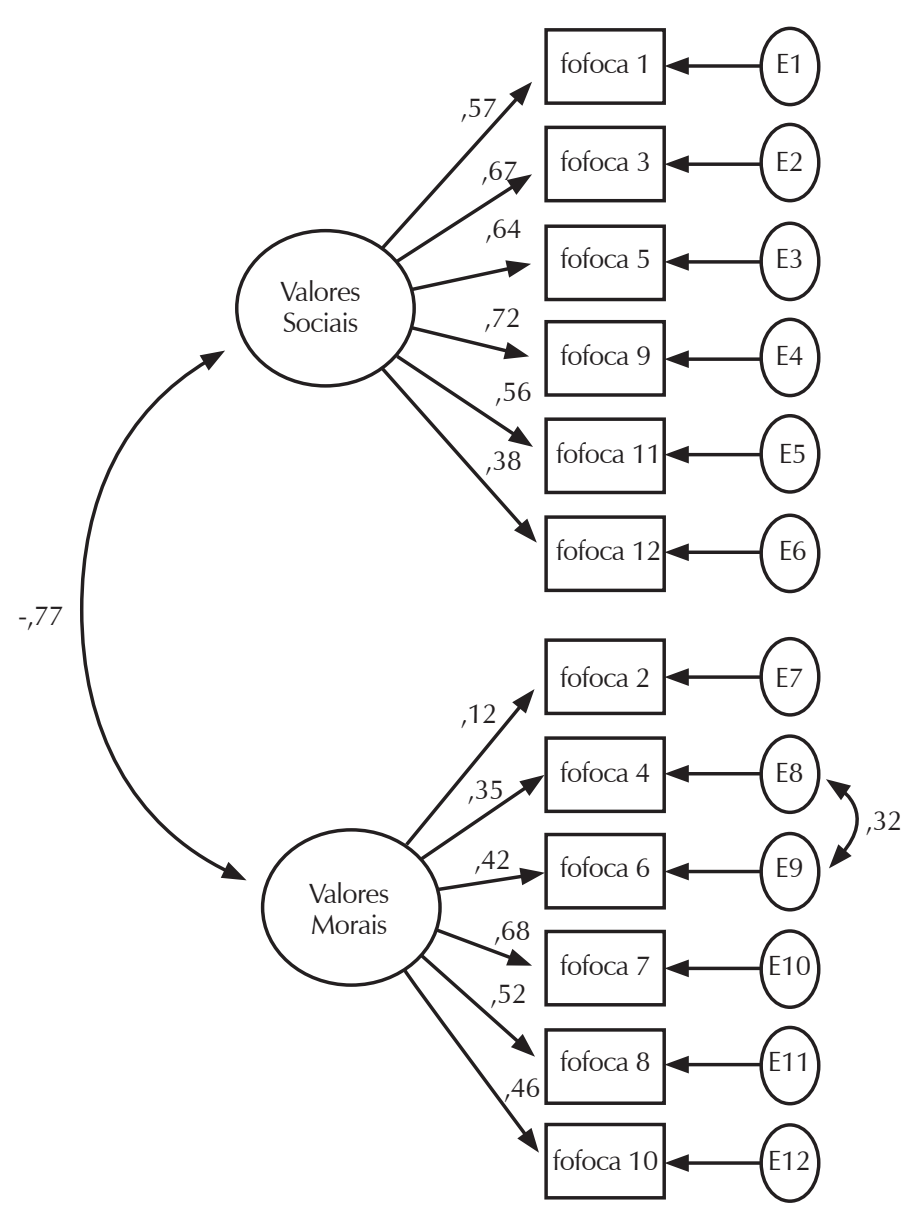

Figura 1. Estrutura fatorial da Escala de Atitudes Frente à Fofoca

Com relação à consistência interna (Alfa de Cronbach, $\alpha$ ) dos fatores da EAFF, verificaram-se os seguintes coeficientes: 0,76 (valor social) e 0,63 (valor moral). A escala como um todo, isto é, o conjunto de doze itens, apresentou consistência interna de 0,78. Para cada fator específico e para a escala total, foi verificado se a retirada de um dos itens poderia aumentar o $\alpha$, o que não ocorreu. Desse modo, mantiveram-se os 6 itens dos fatores de valor social e valor moral. Também foi avaliada a homogeneidade dos fatores, considerando a correlação item-total corrigida. No 
caso do fator valor social, as correlações variaram de 0,32 (item 12) a 0,63 (item 9), sendo o coeficiente médio 0,50 . Já o fator valor moral apresentou correlações entre 0,21 (item 2) e 0,46 (item 6), e coeficiente médio de 0,37 . Todas essas correlações foram estatisticamente diferentes de zero $(r$ $\geq 0,021, p<0,001)$.

\section{Correlatos das atitudes frente à fofoca: evidências de validade discriminante}

Procurando conhecer a influência do sexo nas pontuações dos fatores de atitudes frente à fofoca, isto é, valores sociais e valores morais, realizou-se uma MANOVA. Nesse caso, considerou-se como variável antecedente o sexo, sendo os dois fatores de atitudes frente à fofoca definidos como variáveis-critério. De acordo com os resultados dessa análise, não foi verificado efeito do sexo em relação aos fatores valores sociais (Lambda de Wilks $=0,98, F[1$, 259] $=0,62, p=0,43)$ e valores morais (Lambda de Wilks $=0,98, F[1,259]=$ $3,15, p=0,08)$. Embora não tenham sido encontradas diferenças significativas, cabe destacar que as mulheres $(m=22,4, d p=$ $0,25)$ apresentaram média marginalmente superior aos homens ( $m=21,4, d p=0,42$ ) em valores morais.

Finalmente, foram calculadas as correlações entre as pontuações dos fatores de atitudes frente à fofoca (EAFF) e desejabilidade social (EDSMC). No caso da pontuação total de atitudes frente à fofoca, observouse correlação negativa de moderada para baixa $(r=-0,29, p<0,001)$; o mesmo para a pontuação no fator valores sociais $(r=$ $-0,34, p<0,001)$, porém menos forte para os valores morais $(r=-0,14, p<0,05)$.

\section{Discussão}

O objetivo principal deste estudo foi conhecer evidências de validade e confiabilidade da Escala de Atitudes frente a Fofocas - EAFF (Litman \& Pezzo, 2005) no contexto brasileiro, levando em conta amostra específica de João Pessoa. Confiase que o objetivo tenha sido alcançado; contudo, algumas limitações podem ser levantadas nesta pesquisa, notadamente no que diz respeito aos participantes: tratou-se de amostra não probabilística e específica (estudantes do ensino superior) não numericamente representativa da população brasileira ou mesmo pessoense. Não obstante, tais restrições devem ser ponderadas, uma vez que o propósito não foi generalizar os resultados, mas conhecer as evidências de validade e confiabilidade de uma medida. Discutem-se a seguir os principais resultados.

As evidências de validade fatorial da EAFF parecem bastante consistentes. Coerente com os achados de Litman e Pezzo (2005), foi possível identificar como mais adequado um modelo bifatorial, representado pelos fatores valores sociais e valores morais. Essa estrutura foi mais plausível que aquela que propõe a unifatorialidade dessa medida. Os indicadores de confiabilidade são também coerentes com aqueles descritos por esses autores, sobretudo no que se refere ao fator valor social, que apresentou Alfa de Cronbach muito próximo aos observados em seus Estudos 1 e 2, situando-se acima do ponto de corte 0,70 , que tem sido recomendado na literatura (Nunnally, 1991; Pasquali, 2003). O fator valor moral, porém, apresentou coeficiente inferior àquele verificado por Litman e Pezzo, ficando abaixo de 0,70, porém acima de 0,60, que tem sido aceito para propósitos de pesquisa, principalmente quando o construto avaliado é atitudinal (Mueller, 1986; Peterson, 1994). Os indicadores de homogeneidade reforçam a adequação desse parâmetro psicométrico, uma vez que todas as correlações item-total se situaram acima de 0,20 (Clark \& Watson, 1995).

No que diz respeito ao efeito do sexo nas atitudes frente à fofoca, diferentemente do 
reportado por Baumeister et al. (2004) e Litman e Pezzo (2005), não foi observado qualquer efeito dessa variável. Portanto, apesar de nas pesquisas prévias ter sido indicada a influência do sexo em tais atitudes, com as mulheres tendendo a ser mais favoráveis, no presente estudo, suas médias nos fatores de fofoca não se diferenciaram daquelas dos homens. A direção das pontuações no fator valores morais, no entanto ${ }_{L}$ foi consonante com aquela descrita por Litman e Pezzo (2005), isto é, os homens tendem a considerar a fofoca moralmente mais reprovável do que o fazem as mulheres.

Cabe destacar que a ausência de diferença em relação ao sexo pode dever-se ao fato de a amostra ter sido bastante homogênea, considerando pessoas (estudantes universitários) com focos e interesses bastante parecidos, independentemente de sexo. Uma vez que a fofoca é um comportamento presente em relações sociais e conversas cotidianas (Barkow, 1992; Kniffin \& Wilson, 2005; McPherson, 1991; Noon \& Delbridge, 1993), talvez os universitários se tornem mais parecidos por se depararem com os mesmos contextos. Além disso, tenha-se em conta que existem evidências de que os homens se dedicam mais (transmitindo ou ouvindo) a fofocas sobre celebridades, esportistas e políticos, coerentemente com a visão de serem mais orientados para o ambiente macrossocial, enquanto as mulheres são mais engajadas em fofocas no meio familiar e no círculo de amigos próximos (Ben-Ze'ev, 1994). Contudo, o período de universidade pode significar a mescla de vida pessoal com profissional, tornando os indivíduos bastante iguais nos interesses. Não obstante, essa é meramente uma conjetura. Estudos futuros poderão contribuir para dirimir as dúvidas. Nesse caso, será relevante ampliar a amostra, considerando pessoas da população geral, trabalhadores, donas de casa, aposentados, etc.
A atitude frente à fofoca se correlacionou negativamente com a desejabilidade social, o que está coerente com os achados do Estudo $4 \_$de Litman e Pezzo (2005). A desejabilidade é entendida como um estilo de resposta (viés) ou uma característica de personalidade a partir da qual o respondente tende a dissimular sua resposta real de forma a ser mais aceito socialmente (Gouveia Guerra, Sousa, Santos, \& Costa 2009). Por sua vez, a fofoca pode ser entendida como uma atitude ou um comportamento indesejável, que envolve dizer, predominantemente, coisas ruins ou falsas sobre outras pessoas, $\mathrm{o}$ que pode ser prejudicial (Baumeister et al., 2004). Nesse sentido, esperava-se que esses dois construtos estivessem correlacionados, porém que a correlação entre ambos não fosse tão forte, indicando a validade discriminante da medida de fofocas. De fato, suas medidas compartilharam menos de $12 \%$ da variância $(-0,29 \leq r \leq 0,34)$.

Em resumo, ao adaptar a EAFF para uso no contexto brasileiro reunindo as primeiras evidências de sua validade e confiabilidade, o presente estudo oferece contribuição importante, proporcionando uma medida que permitirá avanços substanciais aos pesquisadores brasileiros interessados nesse tema. Isso também abrirá uma avenida interessante de pesquisas, visando a conhecer os correlatos e o significado atribuído à fofoca como uma atividade social que pode ser importante na vida de muitas pessoas. Uma possibilidade interessante de estudo futuro será conhecer em que medida as atitudes frente à fofoca se correlacionam a construtos sociais (por exemplo, crenças religiosas, ideologias, valores) que têm o papel de inibir comportamentos moral ou socialmente desviantes, como os representados na fofoca. Por fim, como previamente assinalado, é importante diversificar a amostra e o contexto de pesquisa. Talvez valesse a pena, por exemplo, estudar a fofoca no meio laboral, pois ela pode comprometer a estrutura de uma equipe ou dificultar as relações dentro da empresa. 


\section{Valdiney V. Gouveia}

Mestrado em Psicologia Social e do Trabalho pela Universidade de Brasília e Doutorado em Psicologia Social pela Universidade Complutense de Madri. Professor Associado II na Universidade Federal da Paraíba, Paraiba - PB - Brasil.

E-mail:vvgouveia@gmail.com

Deliane Macedo Farias de Sousa

Mestrado em Psicologia pela Universidade Federal do Rio Grande do Norte, Doutoranda em Psicologia Social pela Universidade Federal da Paraíba, Paraíba - PB - Brasil.

E-mail: delianemfs@gmail.com

\section{Andréa Xavier de Albuquerque-Souza}

Mestrado em Psicologia pela Universidade Federal da Paraíba. Doutoranda em Psicologia Social, pela Universidade Federal da Paraíba. Professora do departamento de psicologia da Universidade Estadual da Paraíba e professora da pós graduação da Faculdade de Ciências Sociais Aplicada.

E-mail: andreaxavi@hotmail.com

\section{Roseane Christhina da Nova Sá-Serafim}

Mestre e Doutoranda em Psicologia Social pela Universidade Federal da Paraíba, Professora Assistente I na Unidade Acadêmica de Ciências da Saúde na UFCG, Paraíba - PB - Brasil.

E-mail: psicologiapb@yahoo.com.br

Clênia Maria Toledo de Santana Gonçalves

Mestre em Psicologia Clínica pela Universidade Católica de Pernambuco, e Doutoranda na Universidade Federal da Paraiba, Paraíba, PB - Brasil. E-mail: ctoledosantana@yahoo.com.br

Endereço para envio de correspondência:

Universidade Federal da Paraíba, CCHLA - Departamento de Psicologia, João Pessoa - PB - Brasil. CEP: 58051-900

Recebido 21/7/2010, Aprovado 16/5/2011.

Acheson, J. M. (1988). The lobster gangs of Maine. Hanover, $\mathrm{NH}$ : University of New England Press.

Barkow, J. H. (1992). Beneath new culture is old psychology: Gossip and social stratification. In J. H. Barkow, L. Cosmides, \& J. Tooby (Eds.), The adapted mind (pp. 627637). Oxford, England: Oxford University Press.

Baumeister, R. F., Zhang, L., \& Vohs, K. D. (2004). Gossip as cultural learning. Review of General Psychology, 8, 111121.

Ben-Ze'ev, A. (1994). The vindication of gossip. In R. F. Goodman, \& A. Ben-Ze'ev (Eds.), Good gossip (pp. 1124). Lawrence, KS: University Press of Kansas.

Bergmann, J. R. (1993). Discreet indiscretions: The social organization of gossip. New York: Aldine de Gruyter.

Clark, L. A., \& Watson, D. (1995). Constructing validity: Basic issues in objective scale development. Psychological Assessment,7, 309-319.

Crowne, D., \& Marlowe, D. (1960). A new scale of social desirability independent of psychopathology. Journal of Consulting Psychology, 24, 349-354.

Dunbar, R. I. M. (2004). Gossip in evolutionary perspective. Review of General Psychology, 8, 100-110.

Eder, D., \& Enke, J. L. (1991). The structure of gossip: Opportunities and constraints on collective expression among adolescents. American Sociological Review, 56, 494-508.

Foster, E. K. (2004). Research on gossip: Taxonomy, methods, and future directions. Review of_General Psychology, 8, 78-99.

Garson, G. D. (2003). PA 765 Statnotes: An online textbook. Recuperado em 17 de março de 2010 de http://www2. chass.ncsu.edu/garson/pa765/statnote.htm.

Gouveia, V. V., Guerra, V. M., Sousa, D. M. F. de, Santos, W. S \& Costa, J. de M. (2009). Escala de desejabilidade social de Marlowe-Crowne: Evidências de sua validade fatorial e consistência interna. Avaliação Psicológica, 8, 87-98.

Kniffin, K. M., \& Wilson, D. S. (2005) Utilities of gossip across organizational levels: Multilevel selection, free-riders, and teams. Human Nature, 16, 278-292.
Levin, J., \& Arluke, A. (1987). Gossip: The inside scoop. New York: Plenum.

Litman, J. A., \& Pezzo, M.V. (2005). Individual differences in attitudes towards gossip. Personality and Individual Differences, 38, 963-980.

McAndrew, F. T., \& Milenkovic, M. A. (2002). Of tabloids and family secrets: The evolutionary psychology of gossip. Journal of Applied Social Psychology, 32, 1064-1082.

Mueller, D. J. (1986). Measuring social attitudes: A handbook for researchers and practitioners. New York: Teachers College Press.

Nevo, O, Nevo, B , \& Derech-Zehavi, A. (1994). The tendency to gossip as a psychological disposition: Constructing a measure and validating it. In R. F. Goodman, \& A. BenZe'ev (Eds.), Good gossip (pp. 180-189). Lawrence, KS: University Press of Kansas.

Noon, M., \& Delbridge, R. (1993). News from behind my hand: Gossip in organizations. Organization Studies, 14, 23-36.

Nunnally, J. C. (1991). Teoría psicométrica (E. M. González, trad.). México, DF: Trillas (Trabalho original publicado em 1987).

Paine, R. (1967). What is gossip about? An alternate hypothesis. Man, 2, 278-285.

Pasquali, L. (2003). Psicometria: teoria dos testes na psicologia e na educação. Petrópolis, RJ: Vozes.

Peterson, R. A. (1994). A meta-analysis of Cronbach's coefficient alpha. Journal of Consumer Research, 21, 381-391.

Sabini, J. P., \& Silver, M. (1978). Moral reproach and moral action. Journal for the Theory of Social Behaviour, 8, 103123.

Schein, S. (1994). Used and abused: Gossip in medieval society. In R. F. Goodman, \& A. Ben-Ze'ev (Eds.), Good gossip (pp. 139-153). Lawrence, KS: University of Kansas Press.

Wert, S. R., \& Salovey, P. (2004). A social comparison account of gossip. Review of General Psychology, 8, 122-137. 